\title{
Transverse resistive wall impedances and shielding effectiveness for beam pipes of arbitrary wall thickness
}

\author{
A. M. Al-Khateeb, ${ }^{*}$ R. W. Hasse, O. Boine-Frankenheim, W. M. Daqa, and I. Hofmann \\ FAIR-accelerator theory, GSI-Darmstadt, Planckstrasse 1, D-64291 Darmstadt, Germany
}

(Received 2 April 2007; published 12 June 2007)

\begin{abstract}
Using field matching techniques, closed form analytic expressions for the transverse impedance and for the shielding effectiveness of a smooth cylindrical beam pipe of arbitrary thickness are presented. In the limit of thick and thin beam pipes the well-known expressions are reproduced. The transverse transmission coefficient is compared with the longitudinal one that has been obtained in our previous work [A. M. Al-Khateeb, O. Boine-Frankenheim, R. W. Hasse, and I. Hofmann, Phys. Rev. E 71, 026501 (2005).]. The results are applied to the heavy ion synchrotron SIS 18 and to the planned SIS 100 at GSI. In both machines the stainless steel beam pipe in the dipole sections is much thinner than the skin depths at the revolution frequency and, therefore, the impedance value and the transmission are of concern.
\end{abstract}

DOI: 10.1103/PhysRevSTAB.10.064401

PACS numbers: 29.27.Bd, 29.20.-c, 74.25.Nf

\section{INTRODUCTION}

In large storage rings or synchrotrons, the resistive beam pipe usually represents the main contribution to the real part of the ring impedance at low frequencies. It has been identified as the main driving source for the observed transverse instabilities in the FNAL Recycler [1,2]. In the GSI heavy ion synchrotron SIS 18, the transverse resistivewall instability can be observed during high-current operation [3]. As part of the FAIR accelerator project [4], the SIS 18 presently is upgraded in order to serve as a booster for the projected SIS 100 synchrotron. Because of the low momentum spreads together with high beam intensities and relatively large space-charge tune shifts, the control of coherent beam instabilities induced by the resistive pipe wall will be crucial in both machines [5].

For the design of the SIS 100, accurate expressions for the expected resistive-wall impedance at arbitrary beam energies are needed in order to estimate the growth rates of instabilities and to define the required feedback systems. In the conventional treatment, the resistive-wall impedance is calculated in the ultrarelativistic limit [6,7]. A general formalism for computing impedances of nonrelativistic beams, including resistive-wall boundaries, was given by Gluckstern [8]. In Ref. [9] the resistive-wall impedance for nonrelativistic beams is considered using a direct solution of the Maxwell equations together with an expression for the resulting impedance. For the transverse impedance, the obtained approximation agrees with the result obtained in Ref. [8]. Other recent approaches along these lines can be found in $[10,11]$. In the present work closed form analytic expressions, obtained by applying field matching tech-

\footnotetext{
*Corresponding author. a.alkhateeb@gsi.de

Permanent address: Department of Physics, Yarmouk University, Irbid, Jordan.
}

niques, for arbitrary pipe thickness and beam energy are presented and compared with different approximations.

When the skin depth is larger than the wall thickness, the induced electromagnetic fields can penetrate through the wall and the impedance depends on the structures outside the pipe. In this situation, in addition to the impedance, detailed calculations of the shielding effectiveness of the pipe are necessary, also in order to estimate the currents that could be induced in layers or hardware components behind the pipe, see Ref. [12] and references therein.

The well-known ability of a thin beam pipe of thickness $d$ less than the skin depth $\delta_{s}$ to shield electromagnetic fields excited by beam current modulations was considered in Refs. $[8,12,13]$, where expressions for the impedance and for the shielding effectiveness of thin beam pipes were obtained.

In the present work we consider the transverse impedance and the shielding of the electromagnetic fields excited by transverse dipole oscillations of the beam. The shielding by a beam pipe which is thin as compared to the skin depth is of relevance for the SIS 18 as well as for the design of the SIS 100. The SIS 18 magnets can be ramped with $10 \mathrm{~T} / \mathrm{s}$, the superconducting SIS 100 magnets shall be ramped with $4 \mathrm{~T} / \mathrm{s}$. In order to reduce eddy current effects, the stainless steel beam pipe in the SIS 18 magnets is only $0.3 \mathrm{~mm}$ thick. The skin depth is $1 \mathrm{~mm}$ at the injection energy of $11.4 \mathrm{MeV} / u$. For the stainless steel beam pipe in the new SIS 100 magnets, a wall thickness of a few $0.1 \mathrm{~mm}$ will be required [14]. Because SIS 100 will be a "cold" machine, the heating of the thin pipe due to large image currents can be important. The relevance of this issue for the design of high-current ring machines makes it important to have closed form expressions for the transverse resistive-wall impedance and for the shielding effectiveness covering the relevant ranges of frequencies, beam energies, and wall thicknesses. In the present work these expressions will be derived and compared with approximations. 
The paper is organized as follows: In Sec. II we present the derivation of the dipole source term needed for the calculation of the transverse impedances for a wall of arbitrary thickness. A generalized expression for the longitudinal coupling impedance of an arbitrary azimuthal number $\ell$ will be introduced in Sec. III and then the corresponding transverse coupling impedance will be obtained via the Panofsky-Wenzel theorem. In Sec. IV the impedances and the transmission will be derived by solving the field wave-diffusion equation and by using exact field boundary conditions. The limiting case of a thick wall will be discussed in Sec. V. Using the SIS parameters, we also give in Sec. VI some numerical examples of the dipole transverse resistive-wall impedance as a function of energy $\gamma_{0}$ and frequency $\omega$. Our main conclusions will be presented in Sec. VII.

\section{SOURCE TERM FOR THE TRANSVERSE IMPEDANCE}

Assume a particle beam in form of a circular disk of radius $a$ that moves off axis in the $z$-direction with a constant longitudinal velocity $v=\beta c$ in a cylindrical pipe of radius $b$, where $\beta$ is the relativistic factor and $c$ is the vacuum speed of light. If the beam center is displaced by $\mathbf{d}$, the beam charge density distribution can be represented as follows:

$$
\rho(\mathbf{r}, t)=\sigma(r, \theta) \delta(z-\beta c t)=\frac{Q}{\pi a^{2}} \Theta(a-u) \delta(z-\beta c t),
$$

where $\mathbf{u}=\mathbf{r}-\mathbf{d}, \Theta(\ldots)$ is the Heaviside unit step function, $\delta(\ldots)$ is the Dirac delta function, $Q$ is the total beam charge, and $\sigma(r, \theta)$ is the beam surface charge distribution in the transverse plane. The presence of the displacement $\mathbf{d}$ introduces an additional dependence on the azimuthal angle $\theta$ which in turn will lead to higher multipoles of the beam distribution. The case $\mathbf{d}=0$ corresponds to a beam moving on the axis of the cylindrical beam pipe with a volume charge density $\rho$ given by the following expression:

$$
\rho(\mathbf{r}, t)=\frac{Q}{\pi a^{2}} \Theta(a-r) \delta(z-\beta c t) .
$$

The volume charge density of Eq. (1) for the case $\mathbf{d} \neq 0$ corresponds to an off-axis motion of the kicked beam. In what follows we expand the Heaviside function about $a-$ $r$, viz.

$$
\begin{aligned}
\Theta(a-u)= & \Theta(a-r)+[r-u] \delta(a-r) \\
& +\frac{1}{2}[r-u]^{2} \delta^{\prime}(a-r)+\cdots,
\end{aligned}
$$

where a prime denotes the derivative with respect to the argument. Using the polar representation of $\mathbf{d}$ such that $\mathbf{d}=d\left(\cos \theta_{0} \hat{\mathbf{i}}+\sin \theta_{0} \hat{\mathbf{j}}\right)$, the term $r-u$ takes on the following form for small values of $d$ :

$$
\begin{aligned}
r-u= & r-\sqrt{r^{2}+d^{2}-2 r d \cos \left(\theta-\theta_{0}\right)} \\
= & \frac{d^{4}}{8 r^{3}}-\frac{d^{2}}{2 r}+d\left(1-\frac{d^{2}}{2 r^{2}}\right) \cos \left(\theta-\theta_{0}\right) \\
& +\frac{d^{2}}{2 r} \cos 2\left(\theta-\theta_{0}\right)+\cdots
\end{aligned}
$$

Upon substituting Eq. (4) into (3) and then accounting for terms to first order in the displacement $\mathbf{d}$ such that $d \ll r$ results in the following beam volume charge density:

$$
\begin{aligned}
\rho(r, \theta, z, t) \approx & \frac{Q}{\pi a^{2}} \delta(z-\beta c t)[\Theta(a-r) \\
& \left.+d \cos \left(\theta-\theta_{0}\right) \delta(a-r)\right] .
\end{aligned}
$$

The first two lowest moments of the distribution in Eq. (5) are the total charge $Q$ and the average electric dipole moment $\mathbf{P}=Q \mathbf{d}$ pointing in the direction of the displacement of the beam center. In terms of $P$ and to first order in $d$, the beam volume charge density takes on the following form:

$$
\begin{aligned}
\rho(r, \theta, z, t) \approx & \frac{Q}{\pi a^{2}} \Theta(a-r) \delta(z-\beta c t) \\
& +\frac{P}{\pi a^{2}} \cos \left(\theta-\theta_{0}\right) \delta(a-r) \delta(z-\beta c t) .
\end{aligned}
$$

The first term on the right-hand side of Eq. (6) is the volume charge distribution of the unperturbed (nondisplaced) beam, while the second term accounts for the small movement of the beam center in the direction of $\mathbf{d}$ and it represents a correction or perturbation to the beam volume charge density, namely,

$$
\rho_{1}(r, \theta, z, t)=\frac{P}{\pi a^{2}} \cos \left(\theta-\theta_{0}\right) \delta(a-r) \delta(z-\beta c t) .
$$

The azimuthal dependence in $\rho_{1}$ shows that it is a dipole term and, therefore, it is the source of the dipolar transverse impedance resulting from the off-axis motion of the beam center. Without loss of generality, we assume the beam to be displaced along the $x$-axis such that $\theta_{0}=0$. In the frequency domain, the total volume charge density of Eq. (7) and the corresponding current density take on the following form:

$$
\begin{aligned}
\rho_{1}(r, z, \theta, \omega) & =\frac{P}{\pi a^{2}} \delta(a-r) \cos \theta \frac{e^{i k_{z} z}}{\beta c}, \\
j_{z}(r, z, \theta, \omega) & =\frac{P}{\pi a^{2}} \delta(a-r) \cos \theta e^{i k_{z} z},
\end{aligned}
$$

where $\omega=k_{z} v$ has been used and $k_{z}$ is the wave number in the direction of beam propagation. Equations (8) and (9) are the source terms used by Gluckstern to calculate the transverse impedance for a displaced particle beam [8]. 


\section{TRANSVERSE COUPLING IMPEDANCE AND TRANSVERSE TRANSMISSION}

The Panofsky-Wenzel (PW) theorem relates the transverse force on a charged particle moving parallel to the axis of a cavity to the longitudinal electric field [15]. The theorem gives a relationship between the integrated longitudinal and transverse momentum kicks a particle receives as it traverses (with constant-velocity and in the paraxial limit) an isolated medium or device with an electromagnetic excitation $[6,7,16]$. The PW theorem estimates the transverse impedance from the longitudinal one at the same azimuthal number $\ell$, namely,

$$
Z_{\ell, \perp}^{\text {(total })}(\omega)=\frac{1}{k_{z}} Z_{\ell, \|}^{(\text {total })}(\omega), \quad \ell \neq 0,
$$

where $Z_{\ell, \|}^{\text {(total) }}(\omega)$ and $Z_{\ell, \perp}^{\text {(total) }}(\omega)$ are measured in $\Omega L^{-2 \ell}$ and $\Omega L^{-2 \ell+1}$, respectively. The case $\ell=0$ must be excluded since it corresponds to an on-axis beam motion with zero transverse displacement, see the last paragraph of this section. The transverse Lorentz force will vanish identically for the rotational symmetric mode $\ell=0$ and, hence, particles will not be deflected by the zero azimuthal electromagnetic fields.

We introduce below a generalized expression for the calculation of the longitudinal coupling impedance of an arbitrary azimuthal mode $\ell$ as a volume integral over the corresponding longitudinal electric field within the beam $E_{\ell, z}^{(r \leq a)}(\mathbf{r}, \omega)$ and the source electric current density $j_{z}(\mathbf{r}, \omega)$. Introducing the $\ell$ th electric moment $M_{\ell}$ such that $M_{\ell}=$ $Q d^{\ell}$ with $d$ being the offset from the axis of the beam pipe, we write the following:

$$
\begin{aligned}
Z_{\ell, \|}(\omega)= & \frac{-1}{M_{\ell}^{2}} \int_{0}^{a} \int_{0}^{2 \pi} \int_{0}^{L} E_{\ell, z}^{(r \leq a)}(r, \omega) \\
& \times \cos \ell \theta e^{i k_{z} z} j_{z}^{*}(r, \theta, z, \omega) r d r d \theta d z .
\end{aligned}
$$

The transverse coupling impedance $Z_{\ell, \perp}(\omega)$ is simply obtained from the PW theorem of Eq. (10). For $\ell=0$, Eq. (11) reduces into the well-known expression of the longitudinal coupling impedance of the zero azimuthal measured in $\Omega[6,8,12]$. Using Eq. (11) for $\ell=1$, we obtain the following expressions for the dipole longitudinal coupling impedance $Z_{1, \|}(\omega)$ measured in $\Omega L^{-2}$ and the transverse coupling impedance $Z_{1, \perp}(\omega)$ in $\Omega L^{-1}$, namely,

$$
\begin{aligned}
Z_{1, \|}(\omega)= & \frac{-1}{M_{1}^{2}} \int_{0}^{a} \int_{0}^{2 \pi} \int_{0}^{L} E_{1, z}^{(r \leq a)}(r, \omega) \\
& \times \cos \theta e^{i k_{z} z} j_{z}^{*}(r, \theta, z, \omega) r d r d \theta d z \\
Z_{1, \perp}(\omega)= & \frac{-1}{k_{z} M_{1}^{2}} \int_{0}^{a} \int_{0}^{2 \pi} \int_{0}^{L} E_{1, z}^{(r \leq a)}(r, \omega) \\
& \times \cos \theta e^{i k_{z} z} j_{z}^{*}(r, \theta, z, \omega) r d r d \theta d z .
\end{aligned}
$$

\section{DIPOLAR ELECTROMAGNETIC FIELDS AND IMPEDANCE FOR ARBITRARY WALL THICKNESS}

When the beam pipe has a resistive wall of finite thickness $d=h-b$, the following equations will be used to determine the electric and magnetic fields in each region, namely,

$$
\begin{gathered}
{\left[\frac{d^{2}}{d r^{2}}+\frac{1}{r} \frac{d}{d r}-\frac{\ell^{2}}{r^{2}}-\frac{k_{z}^{2}}{\gamma^{2}}\right] E_{1, z}(r, \omega)} \\
=i \frac{P k_{z} \cos \theta}{\epsilon_{0} \gamma_{0}^{2} \beta c \pi a^{2}} \delta(a-r), \\
{\left[\frac{d^{2}}{d r^{2}}+\frac{1}{r} \frac{d}{d r}-\frac{\ell^{2}}{r^{2}}-\frac{k_{z}^{2}}{\gamma^{2}}\right] B_{1, z}(r, \omega)=0,}
\end{gathered}
$$

where $\gamma^{-2}$ in Eqs. (14) and (15) stands for $\gamma_{0}^{-2}=1-\beta^{2}$ in the nonconducting regions and for $\underline{\gamma}^{-2}=\gamma_{0}^{-2}-$ $i \mu_{0} \omega S / k_{z}^{2}$ within the conducting wall. Here $S$ stands for the conductivity of the wall. Note that Eq. (14) is a homogeneous equation in all regions, except at the beam surface at $r=a$. Introducing $\sigma_{0}$ and $\underline{\sigma}$ such that $\sigma_{0}=k_{z} / \gamma_{0}$ and $\underline{\sigma}=k_{z} / \underline{\gamma}$, the longitudinal field components for the first azimuthal $\ell=1$ become as follows:

$E_{1, z}(r, z, \omega)=e^{i k_{z} z} \begin{cases}A_{1} I_{1}\left(\sigma_{0} r\right) & 0 \leq r \leq a \\ A_{2} I_{1}\left(\sigma_{0} r\right)+A_{3} K_{1}\left(\sigma_{0} r\right) & a \leq r \leq b \\ A_{4} I_{1}(\underline{\sigma} r)+A_{5} K_{1}(\underline{\sigma} r) & b \leq r \leq h \\ A_{6} K_{1}\left(\sigma_{0} r\right) & h \leq r<\infty,\end{cases}$

$B_{1, z}(r, z, \omega)=e^{i k_{z} z} \begin{cases}C_{1} I_{1}\left(\sigma_{0} r\right) & 0 \leq r \leq b \\ C_{2} I_{1}(\underline{\sigma} r)+C_{3} K_{1}(\underline{\sigma} r) & b \leq r \leq h \\ C_{4} K_{1}\left(\sigma_{0} r\right) & h \leq r<\infty\end{cases}$

Upon rewriting the Maxwell equations in cylindrical coordinates, thereby assuming a field variation with $z$ such that $e^{i k_{z} z}$, and writing the Maxwell curl equations in component form, we obtain six scalar equations. Solving for the transverse electromagnetic field components in terms of $E_{1, z}(r, \theta, z, \omega)$ and $B_{1, z}(r, \theta, z, \omega)$, we obtain the following:

$$
\begin{gathered}
E_{1, r}(r, \theta, z, \omega)=-\frac{i \gamma^{2}}{k_{z}^{2}}\left[k_{z} \frac{\partial E_{1, z}}{\partial r}+\frac{\omega}{r} \frac{\partial B_{1, z}}{\partial \theta}\right] \\
E_{1, \theta}(r, \theta, z, \omega)=-\frac{i \gamma^{2}}{k_{z}^{2}}\left[\frac{k_{z}}{r} \frac{\partial E_{1, z}}{\partial \theta}-\omega \frac{\partial B_{1, z}}{\partial r}\right] \\
B_{1, r}(r, \theta, z, \omega)=\frac{i \gamma^{2}}{k_{z}^{2}}\left[\frac{\omega \epsilon_{0} \mu_{0}}{r} \frac{\partial E_{1, z}}{\partial \theta}-k_{z} \frac{\partial B_{1, z}}{\partial r}\right]
\end{gathered}
$$




$$
B_{1, \theta}(r, \theta, z, \omega)=-\frac{i \gamma^{2}}{k_{z}^{2}}\left[\omega \epsilon_{0} \mu_{0} \frac{\partial E_{1, z}}{\partial r}+\frac{k_{z}}{r} \frac{\partial B_{1, z}}{\partial \theta}\right] .
$$

In order to find the integration constants, we need ten boundary conditions. One of these boundary conditions is the discontinuity of $\partial E_{1, z} / \partial r$ at $r=a$. The other nine boundary conditions are the continuity of $E_{1, z}$ at $r=a$, $r=b$, and $r=h$, the continuity of $B_{1, z}, E_{1, \theta}$, and $B_{1, \theta}$ at $r=b$ and $r=h$. Applying the above ten boundary conditions on the interfaces at $r=a, r=b$, and $r=h$, we obtain the following:

$$
\begin{aligned}
E_{1, z}^{(r \leq a)} & =A_{1} I_{1}\left(\sigma_{0} r\right), \quad A_{3}=-i \frac{P \sigma_{0} a I_{1}\left(\sigma_{0} a\right)}{\epsilon_{0} \gamma_{0} \beta c \pi a^{2}}, \\
A_{1}= & \left(\frac{I_{1}(\underline{\sigma} b)}{I_{1}\left(\sigma_{0} b\right)} \frac{\alpha_{22} \beta_{11}-\alpha_{12} \beta_{22}}{\alpha_{11} \alpha_{22}-\alpha_{12} \alpha_{21}}\right. \\
& +\frac{K_{1}(\underline{\sigma} b)}{I_{1}\left(\sigma_{0} b\right)} \frac{\alpha_{11} \beta_{22}-\alpha_{21} \beta_{11}}{\alpha_{11} \alpha_{22}-\alpha_{12} \alpha_{21}}+\frac{K_{1}\left(\sigma_{0} a\right)}{I_{1}\left(\sigma_{0} a\right)} \\
& \left.-\frac{K_{1}\left(\sigma_{0} b\right)}{I_{1}\left(\sigma_{0} b\right)}\right) A_{3} .
\end{aligned}
$$

The constants together with an outline of their derivation can be found in Appendix A. Knowing the longitudinal electric field component of a given azimuthal mode number $\ell$ and the source current density of the mode suffices for finding the corresponding longitudinal impedance measured in units of $\Omega L^{-2 \ell}$. The transverse coupling impedance of the azimuthal $\ell$ can be obtained from the longitudinal one via Eq. (13).

Substituting for $E_{1, z}^{(r \leq a)}(r, \omega)$ and $j_{z}(r, \theta, z, \omega)$ from Eqs. (22) and (9), respectively, we get the following expression for the transverse coupling impedance ( $F$ is dimensionless):

$$
\begin{aligned}
& Z_{1, \|}^{(\text {arbit wall })}(\omega)=i \frac{L I_{1}\left(\sigma_{0} a\right)}{P a} \frac{P \sigma_{0} a I_{1}\left(\sigma_{0} a\right)}{\epsilon_{0} \gamma_{0} \beta c \pi a^{2}} F \\
& =i \frac{n Z_{0}}{2 \beta \gamma_{0}^{2}} \frac{4 I_{1}^{2}\left(\sigma_{0} a\right)}{a^{2}} F \quad\left[\Omega L^{-2}\right] \\
& Z_{1, \perp}^{\text {(arbit wall) }}(\omega)=\frac{Z_{1, \|}^{\text {(arbit wall })}(\omega)}{k_{z}} \\
& =i \frac{n Z_{0}}{2 \beta \gamma_{0}^{2}} \frac{4 I_{1}^{2}\left(\sigma_{0} a\right)}{k_{z} a^{2}} F \quad\left[\Omega L^{-1}\right] \\
& F=\frac{I_{1}(\underline{\sigma} b)}{I_{1}\left(\sigma_{0} b\right)} \frac{\alpha_{22} \beta_{11}-\alpha_{12} \beta_{22}}{\alpha_{11} \alpha_{22}-\alpha_{12} \alpha_{21}}+\frac{K_{1}(\underline{\sigma} b)}{I_{1}\left(\sigma_{0} b\right)} \\
& \times \frac{\alpha_{11} \beta_{22}-\alpha_{21} \beta_{11}}{\alpha_{11} \alpha_{22}-\alpha_{12} \alpha_{21}}+\frac{K_{1}\left(\sigma_{0} a\right)}{I_{1}\left(\sigma_{0} a\right)}-\frac{K_{1}\left(\sigma_{0} b\right)}{I_{1}\left(\sigma_{0} b\right)} .
\end{aligned}
$$

Using the expression for the transverse coupling impedance for arbitrary wall thickness of Eq. (36), the resistivewall part of the transverse impedance is defined as
$Z_{1, \perp}^{(\mathrm{rw})}(\omega)=Z_{1, \perp}^{(\text {arbit wall })}(\omega)-Z_{1, \perp}^{(\mathrm{sc})}(\omega)$. Accordingly, we obtain the following expression for the transverse resistivewall impedance for arbitrary wall thickness:

$$
\begin{aligned}
Z_{1, \perp}^{(\mathrm{rw})}(\omega)= & i \frac{n Z_{0}}{2 \beta \gamma_{0}^{2}} \frac{4 I_{1}^{2}\left(\sigma_{0} a\right)}{k_{z} a^{2}}\left(\frac{I_{1}(\underline{\sigma} b)}{I_{1}\left(\sigma_{0} b\right)} \frac{\alpha_{22} \beta_{11}-\alpha_{12} \beta_{22}}{\alpha_{11} \alpha_{22}-\alpha_{12} \alpha_{21}}\right. \\
& \left.+\frac{K_{1}(\underline{\sigma} b)}{I_{1}\left(\sigma_{0} b\right)} \frac{\alpha_{11} \beta_{22}-\alpha_{21} \beta_{11}}{\alpha_{11} \alpha_{22}-\alpha_{12} \alpha_{21}}\right) .
\end{aligned}
$$

The following well-known approximative resistive-wall formula for a thin wall [17],

$$
Z_{\perp}^{\text {thin wall }}=\frac{L c}{\pi b^{3} d S \omega}
$$

will be used in Sec. VI and its range of validity will be verified by comparison with the exact resistive-wall expression (27).

In the case of a finite wall thickness, we define the electric transmission coefficient as the ratio of the outgoing to incoming radial components of the electric field amplitudes, namely,

$$
\tau_{\perp}=\frac{E_{r}(r=h)}{E_{r}(r=b)}=\frac{\left|A_{4} I^{\prime}(\underline{\sigma} h)+A_{5} K^{\prime}(\underline{\sigma} h)\right|}{\left|A_{4} I^{\prime}(\underline{\sigma} b)+A_{5} K^{\prime}(\underline{\sigma} b)\right|},
$$

where

$$
A_{4}=\frac{\beta_{11} \alpha_{22}-\beta_{22} \alpha_{12}}{\alpha_{11} \alpha_{22}-\alpha_{12} \alpha_{21}}, \quad A_{5}=\frac{\beta_{22} \alpha_{11}-\beta_{11} \alpha_{21}}{\alpha_{11} \alpha_{22}-\alpha_{12} \alpha_{21}}
$$

\section{DIPOLAR ELECTROMAGNETIC FIELDS AND IMPEDANCE FOR A THICK CONDUCTING WALL}

In the limit of a thick beam-pipe conducting wall such that $d \rightarrow \infty$, the general solutions of Eqs. (14) and (15) for the longitudinal electromagnetic field components $E_{1, z}(r, z, \omega)$ and $B_{1, z}(r, z, \omega)$ for $\ell=1$ are

$$
\begin{aligned}
& E_{1, z}(r, z, \omega)=e^{i k_{z} z} \begin{cases}A_{1} I_{1}\left(\sigma_{0} r\right) & 0 \leq r \leq a \\
A_{2} I_{1}\left(\sigma_{0} r\right)+A_{3} K_{1}\left(\sigma_{0} r\right) & a \leq r \leq b \\
A_{4} K_{1}(\underline{\sigma} r) & b \leq r<\infty\end{cases} \\
& B_{1, z}(r, z, \omega)=e^{i k_{z} z} \begin{cases}C_{1} I_{1}\left(\sigma_{0} r\right) & 0 \leq r \leq b \\
C_{2} K_{1}(\underline{\sigma} r) & b \leq r<\infty .\end{cases}
\end{aligned}
$$

In order to find the integration constants, we need six boundary conditions. Integrating the differential equation for $E_{z}$ from $r=a-\delta$ to $r=a+\delta$ for vanishingly small $\delta$, one gets the following boundary condition concerning the discontinuity of $\partial E_{1, z} / \partial r$ at $r=a$, namely,

$$
\frac{\partial E_{1, z}^{r \geq a}}{\partial r}-\frac{\partial E_{1, z}^{r \leq a}}{\partial r}=i \frac{k_{z}}{\epsilon_{0} \gamma_{0}^{2} \beta c} \frac{P}{\pi a^{2}} .
$$


We also use the continuity of $E_{1, z}$ at $r=a$, the continuity of $E_{1, z}, B_{1, z}, E_{1, \theta}$, and $B_{1, \theta}$ at $r=b$. The transverse electromagnetic fields $E_{1, \theta}$ and $B_{1, \theta}$ are directly obtained from Eqs. (19) and (21), respectively. Applying the above six boundary conditions on the interfaces at $r=a$ and $r=b$, the integration constant $A_{1}$ needed for the determination of the electric field inside the beam region and for the calculation of the transverse impedance is given below:

$$
\begin{aligned}
A_{1}= & -i \frac{P k_{z} I_{1}\left(\sigma_{0} a\right)}{\epsilon_{0} \gamma_{0}^{2} \beta c \pi a}\left[\frac{K_{1}\left(\sigma_{0} a\right)}{I_{1}\left(\sigma_{0} a\right)}-\frac{K_{1}\left(\sigma_{0} b\right)}{I_{1}\left(\sigma_{0} b\right)}\right. \\
& \left.+\eta \frac{\gamma_{21}}{\gamma_{22}} \frac{K_{1}(\underline{\sigma} b)}{I_{1}\left(\sigma_{0} b\right)}\right]
\end{aligned}
$$

where $I_{1}^{\prime}$ and $K_{1}^{\prime}$ denote the derivatives of the Bessel functions with respect to the argument. The constants together with an outline of their derivation can be found in Appendix B.

Using the expression for $A_{1}$ above and writing the first azimuthal electric field component $E_{z}(\mathbf{r}, \omega)$ such that $E_{z}(r, \theta, z, \omega)=E_{1, z}(r, \omega) \cos \theta e^{i k_{z} z}$, we obtain the following expression for the electric field in the region $r \leq a$, namely,

$$
\begin{aligned}
E_{1, z}^{(r \leq a)}(r, \omega)= & \frac{-i \omega P I_{1}\left(\sigma_{0} a\right)}{\pi a \epsilon_{0} \gamma_{0}^{2} \beta^{2} c^{2}}\left[\frac{K_{1}\left(\sigma_{0} a\right)}{I_{1}\left(\sigma_{0} a\right)}-\frac{K_{1}\left(\sigma_{0} b\right)}{I_{1}\left(\sigma_{0} b\right)}\right. \\
& \left.+\eta \frac{\gamma_{21}}{\gamma_{22}} \frac{K_{1}(\underline{\sigma} b)}{I_{1}\left(\sigma_{0} b\right)}\right] I_{1}\left(\sigma_{0} r\right) .
\end{aligned}
$$

The corresponding transverse impedance simplifies to

$$
\begin{aligned}
Z_{1, \perp}^{(\text {thick wall })}(\omega)= & \frac{i Z_{0} L I_{1}^{2}\left(\sigma_{0} a\right)}{\pi a^{2} \gamma_{0}^{2} \beta}\left[\frac{K_{1}\left(\sigma_{0} a\right)}{I_{1}\left(\sigma_{0} a\right)}-\frac{K_{1}\left(\sigma_{0} b\right)}{I_{1}\left(\sigma_{0} b\right)}\right. \\
& \left.+\eta \frac{\gamma_{21}}{\gamma_{22}} \frac{K_{1}(\underline{\sigma} b)}{I_{1}\left(\sigma_{0} b\right)}\right] .
\end{aligned}
$$

The third term on the right-hand side of Eq. (36) comes from the coupling between TE and TM modes at the inner surface of the resistive wall. When the beam-pipe wall is perfectly conducting such that $S \rightarrow \infty$ or $\eta \rightarrow 0$, the transverse coupling impedance in Eq. (36) reduces to the following expression [8]:

$$
\begin{aligned}
Z_{1, \perp, \mathrm{TM}+\mathrm{TE}}^{(\mathrm{sc})}(\omega) & =Z_{1, \perp, \mathrm{TM}}^{(\mathrm{sc})}(\omega) \\
& =i \frac{Z_{0} L I_{1}^{2}\left(\sigma_{0} a\right)}{\pi a^{2} \gamma_{0}^{2} \beta}\left[\frac{K_{1}\left(\sigma_{0} a\right)}{I_{1}\left(\sigma_{0} a\right)}-\frac{K_{1}\left(\sigma_{0} b\right)}{I_{1}\left(\sigma_{0} b\right)}\right] .
\end{aligned}
$$

Equation (37) is the well-known transverse space-charge impedance which is directly related to the TM modes of the beam-pipe structure. For a perfectly conducting wall at $r=$ $b$, only TM modes dominantly contribute to the transverse impedance which is a pure space-charge one and capacitive in nature. In this case tangential currents at the wall vanish identically. In the limit $\sigma_{0} a \ll 1$ and $\sigma_{0} b \ll 1$, which usually is referred to as the ultrarelativistic limit, Eq. (37) takes on the following form $[6-8,18,19]$ :

$$
Z_{1, \perp, \mathrm{TM}+\mathrm{TE}}^{(\mathrm{sc})}(\omega)=Z_{1, \perp, \mathrm{TM}}^{(\mathrm{sc})}(\omega)=i \frac{Z_{0} L}{2 \pi \beta \gamma_{0}^{2}}\left[\frac{1}{a^{2}}-\frac{1}{b^{2}}\right] .
$$

The impedance in Eq. (38) can be interpreted as the coherent part of the transverse impedance. However, as can be seen from the arguments of Bessel's functions $\sigma_{0} a$ and $\sigma_{0} b$, namely,

$$
\sigma_{0} a=\frac{a}{\lambda \gamma_{0}} \ll 1, \quad \sigma_{0} b=\frac{b}{\lambda \gamma_{0}} \ll 1, \quad k_{z}=\lambda^{-1},
$$

where $\lambda$ is the wavelength, the limits $\sigma_{0} a \ll 1$ and $\sigma_{0} b \ll$ 1 not only hold for the ultrarelativistic limit $\gamma_{0} \rightarrow \infty$, but also for fixed $\gamma_{0}$ and for $a, b \ll \lambda$. As shown below, the latter condition alone holds for all rings under consideration even for $\gamma_{0}=1$. We see from Eq. (36) that the total transverse coupling impedance is the sum of the pure space-charge impedance of Eq. (37) and a complex part resulting from the wall resistivity. Thus, the resistive-wall contribution to the transverse coupling impedance of a thick wall is

$$
\begin{aligned}
Z_{1, \perp}^{(\mathrm{rw})}(\omega) & =Z_{1, \perp}(\omega)-Z_{1, \perp}^{(\mathrm{sc})}(\omega) \\
& =\frac{i Z_{0} L I_{1}^{2}\left(\sigma_{0} a\right)}{\pi a^{2} \gamma_{0}^{2} \beta} \eta \frac{\gamma_{21}}{\gamma_{22}} \frac{K_{1}(\underline{\sigma} b)}{I_{1}\left(\sigma_{0} b\right)} .
\end{aligned}
$$

The hitherto existing approximative formula for a thick wall [8],

$$
Z_{\perp}^{\text {thickwall }}=(1-i) \frac{\beta_{0} R Z_{0} \delta_{s}}{b^{3}},
$$

where $\delta_{s}=\sqrt{2 / \mu_{0} S \omega}$ is the skin depth at frequency $\omega$, here follows by using the asymptotic expansions of the modified Bessel functions. In the following section, the general transverse resistive-wall impedance Eq. (27) and the transmission coefficient will be evaluated for the parameters of the SIS 18 and SIS 100 synchrotrons.

\section{APPLICATION TO THE SIS 18 AND SIS 100 SYNCHROTRONS}

For the calculation of the impedances and of the transmission coefficients, we provide examples related to SIS 18 and SIS 100 reference parameters given in Table I. The beam pipe in both synchrotrons has variations in geometry and thickness. However, in our examples we will assume a homogenous, round beam pipe. The investigation of the different pipe inhomogeneities is still ongoing. Figure 1 shows the transverse resistive-wall impedance from 
TABLE I. Machine parameters.

\begin{tabular}{lcc}
\hline \hline & SIS 18 & SIS 100 \\
\hline Circumference $L[\mathrm{~m}]$ & 216 & 1080 \\
DC conductivity $S[\Omega \mathrm{m}]^{-1}$ & $10^{6}$ & $10^{6}$ \\
Aperture radius $b[\mathrm{~cm}]$ & 10 & 4 \\
Beam radius $a[\mathrm{~cm}]$ & 5 & 2 \\
Wall thickness $d[\mathrm{~mm}]$ & 0.3 & 0.3 \\
Reference gamma $\gamma_{0}$ & 1.0122 & 1.21 \\
Revolution frequency $f_{0}[\mathrm{kHz}]$ & 214 & 151 \\
\hline \hline
\end{tabular}

Eq. (27) as a function of the wall thickness for SIS 100 parameters. We assume a fixed frequency $f=0.5 f_{0}$ corresponding to the lowest coherent betatron mode at injection energy. The working point range in SIS 100 is still under investigation [20]. For the chosen parameters, the thin wall approximation starts to deviate from the result obtained from Eq. (27) for thicknesses below $d_{\text {SIS }}=$ $0.3 \mathrm{~mm}$. The real part of Eq. (27) reaches a maximum at $d \approx 0.1 \mathrm{~mm}$. For $d \gtrsim \delta_{s}$ the thick wall approximation is reproduced. In Fig. 2 the frequency dependence for a fixed thickness $d=0.3 \mathrm{~mm}$ is shown. The maximum of the real part and the behavior of the impedance at very low frequencies has also been predicted in the frame of an "inductive bypass" model [21].

Finally in Figs. 3 and 4 we plot the transverse transmission coefficient Eq. (29) as a function of the wall thickness and compare it, at the same frequency $f=f_{0}$, with the corresponding longitudinal one derived in Ref. [12]. Figure 3 shows the result for SIS 100 parameters. The transverse transmission vanishes for wall thicknesses larger than the skin thickness $\delta_{s}$, whereas the longitudinal

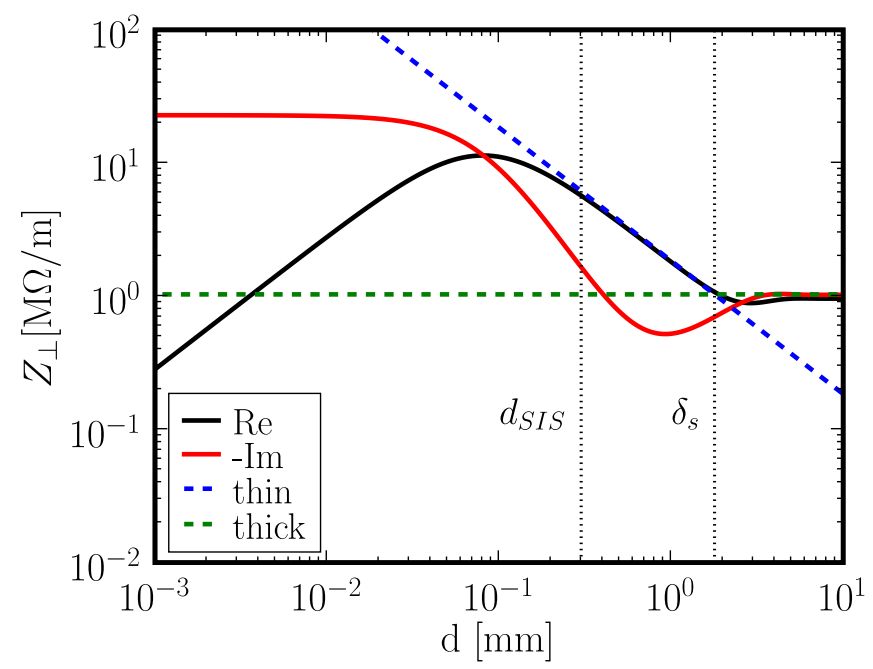

FIG. 1. (Color) The transverse resistive-wall impedance Eq. (27) as a function of the wall thickness for a fixed frequency $f=$ $0.5 f_{0}$ and for SIS 100 parameters. Also shown are the thin and thick wall approximation results. The SIS 100 wall thickness and the skin depth are indicated.

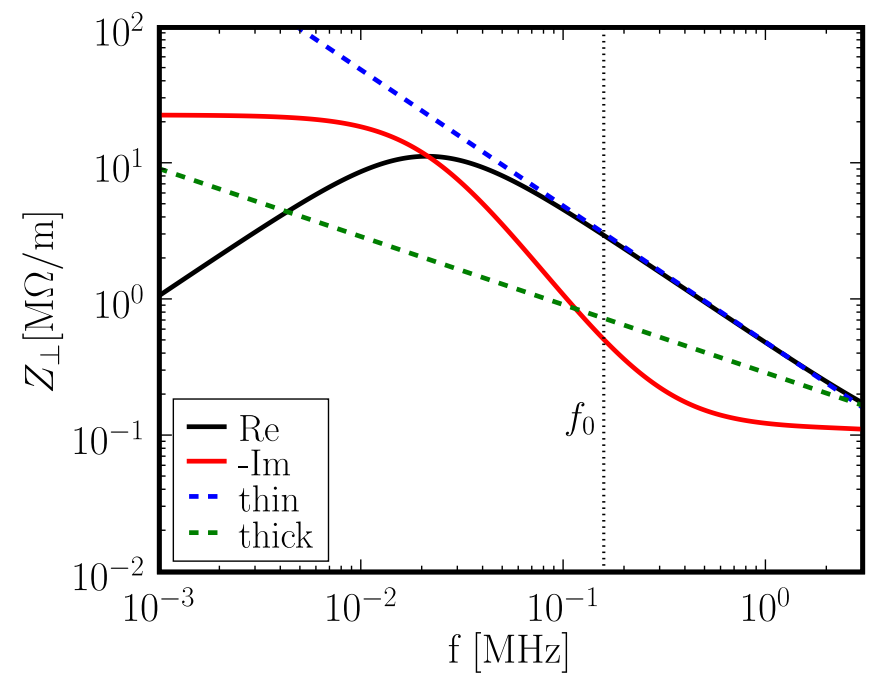

FIG. 2. (Color) The transverse resistive-wall impedance Eq. (27) as a function of the frequency for a fixed wall thickness $d=$ $0.3 \mathrm{~mm}$ and for SIS 100 parameters. Also shown are the thin and thick wall approximation results. The revolution frequency $f_{0}$ at SIS 100 injection is indicated.

shielding factor drops to zero already for much smaller values of $d$. This effect is well known and is due to the fact that longitudinal waves are rather reflected than transmitted, whereas the strong transverse dipole field radiates directly into the radial direction and, hence, contributes to a higher transmission. Here the transmission is dominated by the exponential behavior of the Bessel functions with depth $\underline{\sigma} d$. Figure 4 shows the result for SIS 18, where the difference between the longitudinal and transverse transmission coefficients at injection energy is even more pronounced.

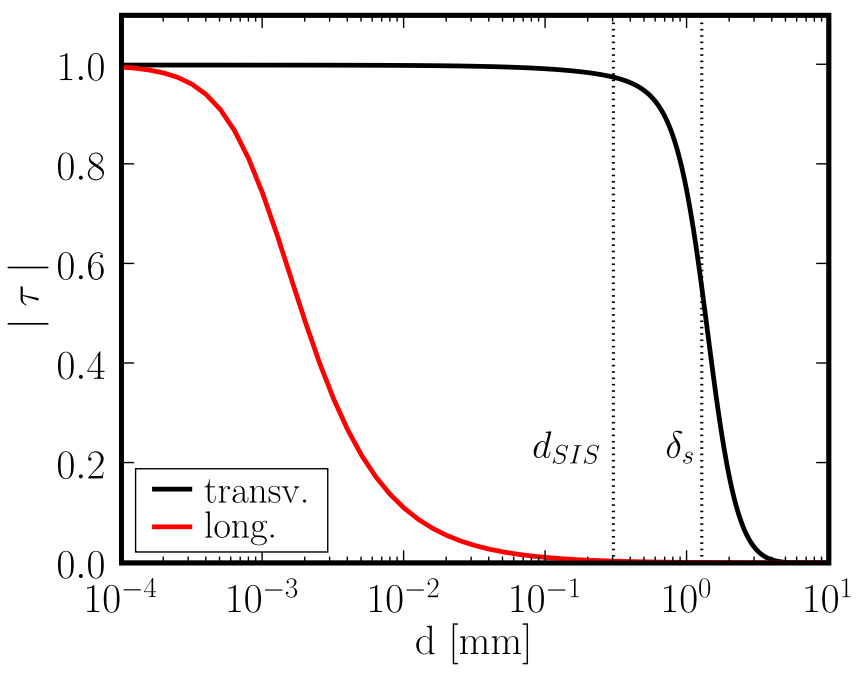

FIG. 3. (Color) Comparison of transverse and longitudinal transmission coefficients for SIS 100 parameters vs wall thickness. The frequency is chosen as $f=0.5 f_{0}$. The wall thickness and the skin depth are indicated. 


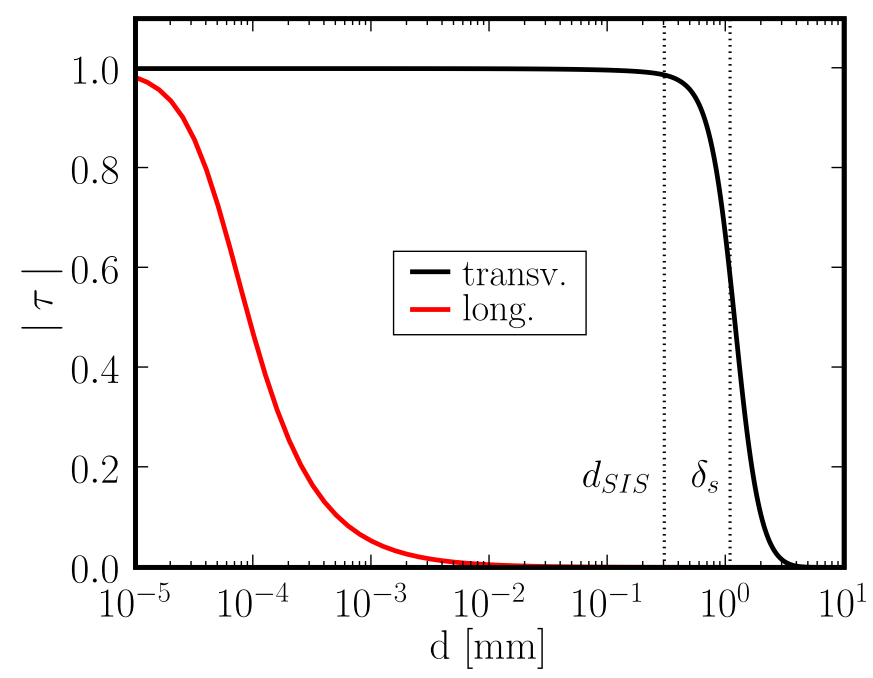

FIG. 4. (Color) Comparison of transverse and longitudinal transmission coefficients for SIS 18 parameters vs wall thickness. The frequency is chosen as $f=f_{0}$. The SIS 18 wall thickness and the skin depth are indicated.

The implication for SIS 100 is, that for the lowest coherent betatron modes the beam pipe in the magnet sections can be regarded as transparent. For the estimation of the transverse impedance, one must therefore include also the components or layers behind the stainless steel wall. The fact that the wall transparency does not necessarily lead to an enhanced impedance is indicated by the measurements of resistive-wall instability growth rates in SIS 18. Here one finds that expression (28) describes quite well the wall impedance at low frequencies [3].

\section{CONCLUSIONS}

Closed form analytic expressions for the transverse impedance and for the shielding effectiveness of a smooth cylindrical beam pipe of arbitrary thickness are presented. The results are compared with the asymptotic expressions for a thin and for a thick wall. For a wall of finite thickness the transmission coefficient is defined based on the radial part of the beam induced electric field entering and leaving the metallic wall. The electric field caused by a transverse dipole oscillations vanishes for a wall thickness comparable to the skin depth. Thinner walls can be regarded as transparent. This is in contrast to the electric field caused by longitudinal perturbations, where wave reflection is dominating and the radial electric field drops already for very small wall thicknesses. In the case of thin beam pipes with $d \leqq \delta_{s}$, as in the SIS 18 and SIS 100 magnet sections, also the layers or structures behind the inner pipe wall can contribute to the impedance seen by the beam.

\section{ACKNOWLEDGMENTS}

A. A. and W.D. thank the FAIR-accelerator theory group, GSI Darmstadt, for their hospitality. They also would like to thank the Council of Scientific Research of Yarmouk University, Irbid, Jordan, for supporting this work by the grant 6/2006. All authors thank Dr. E. Métral, CERN, for many important discussions. The authors acknowledge the support of the European Community Research Infrastructures Action under the FP6 program: Structuring the European Research AreaSpecific Support Action-DESIGN STUDY (Contract No. 515873-DIRACsecondary-Beams).

\section{APPENDIX A: PARAMETERS FOR ARBITRARY WALL THICKNESS}

For the determination of the integration constants, in particular $A_{1}$ needed for the determination of $E_{1, z}$ within the beam, we impose ten boundary conditions. We use the discontinuity of $\partial E_{1, z} / \partial r$ at $r=a$ due the jump discontinuity resulting from $\delta(a-r)$. We also use the continuity of $E_{1, z}$ at $r=a$, and the continuity of $E_{1, z}, B_{1, z}, E_{1, \theta}$, and $B_{1, \theta}$ at $r=b$ and $r=h$. Applying the above ten boundary conditions on the interfaces at $r=a, r=b$, and $r=h$, we obtain the following algebraic equations:

$$
\begin{aligned}
& A_{1} I_{1}\left(\sigma_{0} a\right)=A_{2} I_{1}\left(\sigma_{0} a\right)+A_{3} K_{1}\left(\sigma_{0} a\right) \\
& A_{2} I_{1}^{\prime}\left(\sigma_{0} a\right)+A_{3} K_{1}^{\prime}\left(\sigma_{0} a\right)-A_{1} I_{1}^{\prime}\left(\sigma_{0} a\right) \\
& \quad=i \frac{P}{\epsilon_{0} \gamma_{0} \beta c \pi a^{2}} \equiv i f_{0}
\end{aligned}
$$

$$
\begin{gathered}
A_{2} I_{1}\left(\sigma_{0} a\right)+A_{3} K_{1}\left(\sigma_{0} a\right)=A_{4} I_{1}(\underline{\sigma} b)+A_{5} K_{1}(\underline{\sigma} b) \\
A_{4} I_{1}(\underline{\sigma} b)+A_{5} K_{1}(\underline{\sigma} b)=A_{6} K_{1}\left(\sigma_{0} h\right) \\
-\eta\left[A_{2} I_{1}^{\prime}\left(\sigma_{0} b\right)+A_{3} K_{1}^{\prime}\left(\sigma_{0} b\right)\right]+A_{4} I_{1}^{\prime}(\underline{\sigma} b)+A_{5} K_{1}^{\prime}(\underline{\sigma} b) \\
=\eta \frac{\omega}{k_{z} \sigma_{0} b} C_{1} I_{1}\left(\sigma_{0} b\right)-\frac{\omega}{k_{z} \underline{\sigma} b}\left[C_{2} I_{1}(\underline{\sigma} b)+C_{3} K_{1}(\underline{\sigma} b)\right]
\end{gathered}
$$

$$
\begin{gathered}
A_{4} I_{1}^{\prime}(\underline{\sigma} h)+A_{5} K_{1}^{\prime}(\underline{\sigma} h)+\frac{\omega}{k_{z} \underline{\sigma} h}\left[C_{2} I_{1}(\underline{\sigma} h)+C_{3} K_{1}(\underline{\sigma} h)\right] \\
\quad=\eta A_{6} K_{1}^{\prime}\left(\sigma_{0} h\right)+\frac{\omega}{k_{z} \sigma_{0} h} C_{4} K_{1}\left(\sigma_{0} h\right)
\end{gathered}
$$

$$
C_{1} I_{1}\left(\sigma_{0} b\right)=C_{2} I_{1}(\underline{\sigma} b)+C_{3} K_{1}(\underline{\sigma} b)
$$




$$
\begin{aligned}
C_{4} K_{1}\left(\sigma_{0} h\right) & =C_{2} I_{1}(\underline{\sigma} h)+C_{3} K_{1}(\underline{\sigma} h) \\
\eta & =\frac{\omega \epsilon_{0} \gamma_{0}}{i \underline{\gamma}\left(S-i \omega \epsilon_{0}\right)}
\end{aligned}
$$

$$
\begin{gathered}
\frac{i \gamma_{0}^{2}}{k_{z} b}\left[A_{2} I_{1}\left(\sigma_{0} b\right)+A_{3} K_{1}\left(\sigma_{0} b\right)\right]+\frac{i \gamma_{0} \omega}{k_{z}} C_{1} I_{1}^{\prime}\left(\sigma_{0} b\right) \\
=\frac{i \underline{\gamma}^{2}}{k_{z} b}\left[A_{4} I_{1}(\underline{\sigma} b)+A_{5} K_{1}(\underline{\sigma} b)\right] \\
+\frac{i \underline{\gamma} \omega}{k_{z}}\left[C_{2} I_{1}^{\prime}(\underline{\sigma} b)+C_{3} K_{1}^{\prime}(\underline{\sigma} b)\right]
\end{gathered}
$$$$
\frac{i \underline{\gamma}^{2}}{k_{z} h}\left[A_{4} I_{1}(\underline{\sigma} h)+A_{5} K_{1}(\underline{\sigma} h)\right]
$$$$
+\frac{i \underline{\gamma} \omega}{k_{z}}\left[C_{2} I_{1}^{\prime}(\underline{\sigma} h)+C_{3} K_{1}^{\prime}(\underline{\sigma} h)\right]
$$$$
=\frac{i \gamma_{0}^{2}}{k_{z} h} A_{6} K_{1}\left(\sigma_{0} h\right)+\frac{i \gamma_{0} \omega}{k_{z}} C_{4} K_{1}^{\prime}\left(\sigma_{0} h\right)
$$

Upon solving the above equations simultaneously, we obtain the following:

$$
\begin{gathered}
E_{1, z}^{(r \leq a)}=A_{1} I_{1}\left(\sigma_{0} r\right), \quad A_{3}=-i \frac{P \sigma_{0} a I_{1}\left(\sigma_{0} a\right)}{\epsilon_{0} \gamma_{0} \beta c \pi a^{2}}, \\
f_{00}=1-\frac{\gamma^{2}}{\gamma_{0}^{2}} \\
f_{3}=\frac{K_{1}(\underline{\sigma} h)}{I_{1}(\underline{\sigma} h)}-\frac{K_{1}(\underline{\sigma} b)}{I_{1}(\underline{\sigma} b)}, \quad a_{00}=\frac{\omega}{k_{z} \underline{\sigma} h}\left(1-\frac{\eta_{3} \underline{\sigma}}{\sigma_{0}}\right), \\
\eta_{3}=\frac{\omega \epsilon_{0} \gamma_{0}}{i \underline{\gamma}\left(S_{w}-i \omega \epsilon_{w}\right)}
\end{gathered}
$$

$$
\begin{aligned}
A_{1}= & \left(\frac{I_{1}(\underline{\sigma} b)}{I_{1}\left(\sigma_{0} b\right)} \frac{\alpha_{22} \beta_{11}-\alpha_{12} \beta_{22}}{\alpha_{11} \alpha_{22}-\alpha_{12} \alpha_{21}}+\frac{K_{1}(\underline{\sigma} b)}{I_{1}\left(\sigma_{0} b\right)}\right. \\
& \left.\times \frac{\alpha_{11} \beta_{22}-\alpha_{21} \beta_{11}}{\alpha_{11} \alpha_{22}-\alpha_{12} \alpha_{21}}+\frac{K_{1}\left(\sigma_{0} a\right)}{I_{1}\left(\sigma_{0} a\right)}-\frac{K_{1}\left(\sigma_{0} b\right)}{I_{1}\left(\sigma_{0} b\right)}\right) A_{3}
\end{aligned}
$$

$$
\alpha_{11}=c_{11} \frac{I_{1}(\underline{\sigma} b)}{I_{1}\left(\sigma_{0} b\right)}+c_{21}-\eta_{3} \frac{K_{1}^{\prime}\left(\sigma_{0} h\right)}{K_{1}\left(\sigma_{0} h\right)} \frac{a_{11}}{a_{00}} \frac{I_{1}(\underline{\sigma} h)}{K_{1}\left(\sigma_{0} h\right)}
$$

$$
\alpha_{12}=c_{11} \frac{K_{1}(\underline{\sigma} b)}{I_{1}\left(\sigma_{0} b\right)}+c_{22}-\eta_{3} \frac{K_{1}^{\prime}\left(\sigma_{0} h\right)}{K_{1}\left(\sigma_{0} h\right)} \frac{a_{11}}{a_{00}} \frac{K_{1}(\underline{\sigma} h)}{K_{1}\left(\sigma_{0} h\right)}
$$

$$
\beta_{11}=c_{11} \frac{K_{1}\left(\sigma_{0} b\right)}{I_{1}\left(\sigma_{0} b\right)}-c_{12}, \quad \beta_{22}=e_{11} \frac{K_{1}\left(\sigma_{0} b\right)}{I_{1}\left(\sigma_{0} b\right)}-e_{33}
$$

$$
\begin{aligned}
& \alpha_{21}=e_{11} \frac{I_{1}(\underline{\sigma} b)}{I_{1}\left(\sigma_{0} b\right)}-d_{11}-d_{21} \frac{I_{1}(\underline{\sigma} h)}{K_{1}\left(\sigma_{0} h\right)}, \\
& \alpha_{22}=e_{11} \frac{K_{1}(\underline{\sigma} b)}{I_{1}\left(\sigma_{0} b\right)}-d_{12}-d_{21} \frac{K_{1}(\underline{\sigma} h)}{K_{1}\left(\sigma_{0} h\right)}
\end{aligned}
$$

$$
\begin{aligned}
& c_{11}=f_{00} f_{3} I_{1}\left(\sigma_{0} b\right)-\eta_{3} \frac{b}{h} \frac{I_{1}^{\prime}\left(\sigma_{0} b\right)}{I_{1}\left(\sigma_{0} b\right)} \frac{a_{12}}{a_{00}}, \\
& c_{12}=f_{00} f_{3} K_{1}\left(\sigma_{0} b\right)-\eta_{3} \frac{b}{h} \frac{K_{1}^{\prime}\left(\sigma_{0} b\right)}{I_{1}\left(\sigma_{0} b\right)} \frac{a_{12}}{a_{00}}
\end{aligned}
$$

$$
e_{11}=\frac{\eta_{3} a_{22}}{a_{00}} \frac{b}{h} \frac{I_{1}^{\prime}\left(\sigma_{0} b\right)}{I_{1}\left(\sigma_{0} b\right)}, \quad e_{33}=\frac{\eta_{3} a_{22}}{a_{00}} \frac{b}{h} \frac{K_{1}^{\prime}\left(\sigma_{0} b\right)}{I_{1}\left(\sigma_{0} b\right)}
$$

$$
\begin{aligned}
a_{11}= & \frac{\gamma b \omega}{\gamma_{0}^{2}} K_{1}^{\prime}(\underline{\sigma} b) \frac{K_{1}\left(\sigma_{0} h\right)}{I_{1}(\underline{\sigma} h)}-\frac{\gamma}{\gamma_{0}^{2}} I_{1}^{\prime}(\underline{\sigma} b) \frac{K_{1}(\underline{\sigma} b)}{I_{1}(\underline{\sigma} b)} \\
& \times \frac{K_{1}\left(\sigma_{0} h\right)}{I_{1}(\underline{\sigma} h)}
\end{aligned}
$$




$$
\begin{gathered}
a_{12}=\frac{\underline{\gamma} b \omega}{\gamma_{0}^{2}} I_{1}^{\prime}(\underline{\sigma} b) \frac{K_{1}(\underline{\sigma} h)}{I_{1}(\underline{\sigma} h)} \frac{I_{1}\left(\sigma_{0} b\right)}{I_{1}(\underline{\sigma} b)}-\frac{\underline{\gamma} b \omega}{\gamma_{0}^{2}} K_{1}^{\prime}(\underline{\sigma} b) \frac{I_{1}\left(\sigma_{0} b\right)}{I_{1}(\underline{\sigma} b)} \\
-\frac{b \omega}{\gamma_{0}} I_{1}^{\prime}\left(\sigma_{0} b\right) f_{3} \\
a_{21}=\frac{\underline{\gamma} h \omega}{\gamma_{0}^{2}} K_{1}^{\prime}(\underline{\sigma} h) \frac{K_{1}\left(\sigma_{0} h\right)}{I_{1}(\underline{\sigma} h)}-\frac{\underline{\gamma} h \omega}{\gamma_{0}^{2}} I_{1}^{\prime}(\underline{\sigma} h) \frac{K_{1}(\underline{\sigma} b)}{I_{1}(\underline{\sigma} b)} \\
\times \frac{K_{1}\left(\sigma_{0} h\right)}{I_{1}(\underline{\sigma} h)}-\frac{h \omega}{\gamma_{0}} K_{1}^{\prime}\left(\sigma_{0} h\right) f_{3} \\
a_{22}=\frac{\underline{\gamma} h \omega}{\gamma_{0}^{2}} I_{1}^{\prime}(\underline{\sigma} h) \frac{K_{1}(\underline{\sigma} h)}{I_{1}(\underline{\sigma} h)} \frac{I_{1}\left(\sigma_{0} b\right)}{I_{1}(\underline{\sigma} b)} \\
-\frac{\gamma h \omega}{\gamma_{0}^{2}} K_{1}^{\prime}(\underline{\sigma} h) \frac{I_{1}\left(\sigma_{0} b\right)}{I_{1}(\underline{\sigma} b)} .
\end{gathered}
$$

\section{APPENDIX B: PARAMETERS FOR A THICK WALL}

For the determination of the integration constants, in particular $A_{1}$ needed for the determination of $E_{1, z}$ within the beam, we impose six boundary conditions. We use the discontinuity of $\partial E_{1, z} / \partial r$ at $r=a$ due the jump discontinuity resulting from $\delta(a-r)$. We also use the continuity of $E_{1, z}$ at $r=a$, and the continuity of $E_{1, z}, B_{1, z}, E_{1, \theta}$, and $B_{1, \theta}$ at $r=b$. Applying the above six boundary conditions on the interfaces at $r=a$ and $r=b$, we obtain the following algebraic equations:

$$
\begin{gathered}
A_{1} I_{1}\left(\sigma_{0} a\right)=A_{2} I_{1}\left(\sigma_{0} a\right)+A_{3} K_{1}\left(\sigma_{0} a\right) \\
A_{2} I_{1}^{\prime}\left(\sigma_{0} a\right)+A_{3} K_{1}^{\prime}\left(\sigma_{0} a\right)-A_{1} I_{1}^{\prime}\left(\sigma_{0} a\right) \\
=i \frac{P}{\epsilon_{0} \gamma_{0} \beta c \pi a^{2}} \equiv i f_{0} \\
-\eta\left[A_{2} I_{1}^{\prime}\left(\sigma_{0} b\right)+A_{3} K_{1}^{\prime}\left(\sigma_{0} b\right)\right]+A_{4} K_{1}^{\prime}(\underline{\sigma} b) \\
=\eta \frac{\omega}{k_{z} \sigma_{0} b} C_{1} I_{1}\left(\sigma_{0} b\right)-\frac{\omega}{k_{z} \underline{\sigma} b} C_{2} K_{1}(\underline{\sigma} b) \\
C_{1} I_{1}(\underline{\sigma} b)=C_{2} K_{1}(\underline{\sigma} b), \quad \eta=\frac{\omega \epsilon_{0} \gamma_{0}}{i \underline{\gamma}\left(S-i \omega \epsilon_{0}\right)} \\
\frac{\omega}{k_{z} \sigma_{0} b} C_{1} I_{1}\left(\sigma_{0} b\right)=A_{4} K_{1}(\underline{\sigma} b) \\
\frac{i \gamma_{0}^{2}}{k_{z} b}\left[A_{2} I_{1}\left(\sigma_{0} b\right)+A_{3} K_{1}\left(\sigma_{0} b\right)\right]+\frac{i \gamma_{0} \omega}{k_{z}} C_{1} I_{1}^{\prime}\left(\sigma_{0} b\right) \\
=\frac{i \underline{\gamma^{2}}}{k_{z} b} A_{4} K_{1}(\underline{\sigma} b)+\frac{i \underline{\gamma} \omega}{k_{z}} C_{2} K_{1}^{\prime}(\underline{\sigma} b),
\end{gathered}
$$

where $I^{\prime}$ and $K^{\prime}$ mean the derivatives with respect to the argument. Upon solving for $A_{4}$ and $C_{2}$ and substituting for $A_{3}$, we obtain the following:

$$
\begin{aligned}
& A_{4}=-i \frac{P k_{z} I_{1}\left(\sigma_{0} a\right)}{\epsilon_{0} \gamma_{0}^{2} \beta c \pi a} \frac{\eta \gamma_{21}}{\gamma_{22}}, \\
& A_{2}=- i \frac{P k_{z} I_{1}\left(\sigma_{0} a\right)}{\epsilon_{0} \gamma_{0}^{2} \beta c \pi a}\left[\eta \frac{\gamma_{21}}{\gamma_{22}} \frac{K_{1}(\underline{\sigma} b)}{I_{1}\left(\sigma_{0} b\right)}-\frac{K_{1}\left(\sigma_{0} b\right)}{I_{1}\left(\sigma_{0} b\right)}\right] \\
& C_{2}=-i \frac{P k_{z} I_{1}\left(\sigma_{0} a\right)}{\epsilon_{0} \gamma_{0}^{2} \beta c \pi a} \frac{\eta \gamma_{21}}{\gamma_{22}} \frac{\gamma_{0}^{2}-\underline{\gamma^{2}}}{\underline{\gamma} b \gamma_{11}} \\
& C_{1}=-i \frac{P k_{z} I_{1}\left(\sigma_{0} a\right)}{\epsilon_{0} \gamma_{0}^{2} \beta c \pi a} \frac{\eta \gamma_{21}}{\gamma_{22}} \frac{\gamma_{0}^{2}-\underline{\gamma} \underline{\gamma} \gamma_{11}}{I_{1}\left(\sigma_{0} b\right)} \\
& A_{1}=-i \frac{P k_{z} I_{1}\left(\sigma_{0} a\right)}{\epsilon_{0} \gamma_{0}^{2} \beta c \pi a}\left[\frac{K_{1}\left(\sigma_{0} a\right)}{I_{1}\left(\sigma_{0} a\right)}-\frac{K_{1}\left(\sigma_{0} b\right)}{I_{1}\left(\sigma_{0} b\right)}\right. \\
&\left.+\eta \frac{\gamma_{21}}{\gamma_{22}} \frac{K_{1}(\underline{\sigma} b)}{I_{1}\left(\sigma_{0} b\right)}\right], \\
& A_{3}=-i f_{0} \sigma_{0} a I_{1}\left(\sigma_{0} a\right) \\
& \gamma_{12}= \frac{K_{1}^{\prime}(\underline{\sigma} b)}{K_{1}(\underline{\sigma} b)}-\eta \frac{I_{1}^{\prime}\left(\sigma_{0} b\right)}{I_{1}\left(\sigma_{0} b\right)}, \\
& \gamma_{21}= \frac{K_{1}^{\prime}\left(\sigma_{0} b\right)}{K_{1}(\underline{\sigma} b)}-\frac{I_{1}^{\prime}\left(\sigma_{0} b\right)}{I_{1}\left(\sigma_{0} b\right)} \frac{K_{1}\left(\sigma_{0} b\right)}{K_{1}(\underline{\sigma} b)} \\
& \gamma_{11}=\frac{K_{1}^{\prime}(\underline{\sigma} b)}{K_{1}(\underline{\sigma} b)}-\frac{\gamma_{0}}{\underline{\gamma}} \frac{I_{1}^{\prime}\left(\sigma_{0} b\right)}{I_{1}\left(\sigma_{0} b\right)}, \\
& \gamma_{22}=\gamma_{12}+\left(1-\eta \frac{\gamma}{\gamma}\right) \frac{\gamma_{0}^{2}-\underline{\gamma^{2}}}{k_{z}^{2} b^{2} \gamma_{11}} .
\end{aligned}
$$

Using the expression for $A_{1}$ above, we obtain the following expression for the electric field in the region $r \leq a$, namely,

$$
\begin{aligned}
E_{1, z}(r, \theta, z, \omega)= & \frac{-i \omega P I_{1}\left(\sigma_{0} a\right) \cos \theta e^{i k_{z} z}}{\pi a \epsilon_{0} \gamma_{0}^{2} \beta^{2} c^{2}}\left[\frac{K_{1}\left(\sigma_{0} a\right)}{I_{1}\left(\sigma_{0} a\right)}\right. \\
& \left.-\frac{K_{1}\left(\sigma_{0} b\right)}{I_{1}\left(\sigma_{0} b\right)}+\eta \frac{\gamma_{21}}{\gamma_{22}} \frac{K_{1}(\underline{\sigma} b)}{I_{1}\left(\sigma_{0} b\right)}\right] I_{1}\left(\sigma_{0} r\right) .
\end{aligned}
$$

[1] V. Lebedev and A. Burov, AIP Conf. Proc. 773, 350 (2005).

[2] K. Y. Ng, AIP Conf. Proc. 773, 365 (2005).

[3] V. Kornilov, O. Boine-Frankenheim, and I. Hofmann, Proceedings of the 39th ICFA Advanced Beam Dynamics Workshop, 2006, Tsukuba, Japan.

[4] W. Henning, Proceedings of EPAC 2004, Lucerne, p. 50.

[5] O. Boine-Frankenheim, Proceedings of EPAC 2006, Edinburgh, p. 1886. 
[6] B. W. Zotter and S. A. Kheifets, Impedances and Wakes in High-Energy Particle Accelerators (World Scientific, Singapore, 1998), Chap. 6.

[7] A. W. Chao, Physics of Collective Beam Instabilities in High Energy Accelerators (Wiley, New York, 1993).

[8] R. L. Gluckstern, CERN Report No. 2000-011 (2000), p. 9.

[9] F. Zimmermann and K. Oide, Phys. Rev. ST Accel. Beams 7, 044201 (2004).

[10] B. Zotter, CERN Report No. CERN-AB-043.

[11] E. Métral, CERN Report No. CERN-AB-084.

[12] A. M. Al-Khateeb, O. Boine-Frankenheim, R. W. Hasse, and I. Hofmann, Phys. Rev. E 71, 026501 (2005).

[13] R. L. Gluckstern and B. Zotter, Phys. Rev. ST Accel. Beams 4, 024402 (2001).

[14] S. Wilfert and K. Keutel, Die Kryogenen Vakuumkammern der Supraleitenden Magnete der Synchrotrons SIS100/ 300, internal report, GSI Darmstadt, 2004.

[15] W. Panofsky and W. Wenzel, Rev. Sci. Instrum. 27, 967 (1956).

[16] King-Yuen Ng, Fermi Report No. FN-443, 1987.

[17] J.-L. Laclare, in Proceedings of the CERN Accelerator School: 5th General Accelerator Physics Course, 1992, Jyväskylä, Finland (CERN-94-01, Vol. 1).

[18] L. Palumbo, V. G. Vaccaro, and M. Zubov, LNF-94/041 (P), 1994.

[19] J.-L. Laclare, CERN Report No. 85-19, 1985, pp. 377414.

[20] G. Franchetti, O. Boine-Frankenheim, I. Hofmann, V. Kornilov, P. Spiller, and J. Stadlmann, Proceedings of EPAC 2006, p. 2793.

[21] L. Vos, CERN-AB-2003-005, 2003. 